\title{
POTENCIAL DA MACROFAUNA E OUTRAS VARIÁVEIS EDÁFICAS COMO INDICADORES DA QUALIDADE DO SOLO EM ÁREAS COM ARAUCARIA ANGUSTIFOLIA
}

\author{
Dilmar BARETTA, ${ }^{1 *}$ George Gardner BROWN² \& Elke Jurandy Bran Nogueira \\ CARDOSO $^{3}$ \\ ${ }^{1}$ Programa de Pós-Graduação em Solos e Nutrição de Plantas, Escola Superior de Agricultura "Luiz \\ de Queiroz" (ESALQ/USP). Avenida Pádua Dias, 11, CEP 13418-900, Piracicaba, São Paulo, Brasil. \\ E-mail: baretta@udesc.br \\ ${ }^{2}$ Embrapa Florestas. Estrada da Ribeira, km 111, CEP 83411-000, Curitiba, Paraná, Brasil. E-mail: \\ browng@cnpf.embrapa.br \\ ${ }^{3}$ Departamento de Ciência do Solo, Escola Superior de Agricultura "Luiz de Queiroz" (ESALQ/USP). \\ Avenida Pádua Dias, 11, CEP 13418-900, Piracicaba, São Paulo, Brasil. \\ E-mail: ejbncard@esalq.usp.br
}

Baretta, D., G. G. Brown \& E. J. B. Nogueira Cardoso. 2010. Potencial da macrofauna e outras variáveis edáficas como indicadores da qualidade do solo em áreas com Araucaria angustifolia. Acta Zoológica Mexicana (n.s.), Número Especial 2: 135-150.

RESUMO. O estudo da macrofauna do solo em áreas com Araucaria angustifolia é importante para entender os processos edáficos que ocorrem nestes ecossistemas, já que esses animais atuam na decomposição e mineralização da matéria orgânica e na manutenção da estrutura do solo. O presente estudo teve o objetivo de avaliar, em áreas com araucária naturais e reflorestadas, impactadas ou não pela queima acidental, o potencial indicador da macrofauna edáfica e de variáveis ambientais (químicas e microbiológicas do solo) para separar as áreas, por meio da análise canônica discriminante (ACD). As áreas estudadas incluíram: floresta nativa com araucária, reflorestamento de araucária, reflorestamento de araucária submetido a incêndio acidental, e campo nativo com araucárias nativas e ocorrência de incêndio. Em cada área, dez amostras de solo foram coletadas, próximo a dez árvores de araucária selecionadas ao acaso, em três épocas contrastantes, usando o método de escavação e triagem manual de monólitos (TSBF). Observou-se um impacto importante da intervenção antrópica sobre os atributos biológicos e químicos do solo. A macrofauna edáfica apresentou potencial para ser usada como indicadora da qualidade do solo. Os grupos Diplopoda, Chilopoda, Isoptera e Araneae, e biomassa da macrofauna, índice de diversidade de Shannon $(\mathrm{H})$, matéria seca total da serapilheira, fósforo e atributos microbiológicos, especialmente carbono da biomassa microbiana e respiração basal foram os responsáveis por praticamente toda a separação entre as áreas, sendo bons indicadores das modificações que ocorreram nos ecossistemas. A contribuição de cada atributo para separar as vieras teve efeito da sazonalidade.

Palavras chave: Biodiversidade, análise multivariada, análise canônica discriminante, impactos ambientais, fogo acidental, minhocas.

*Endereçoatual:LaboratóriodeSolos(CEO/UDESC),R.BeloniTrombetaZanin,680-E,Chapecó,SC,89806-070,Brasil. Recibido: 16/05/2008; aceptado: 08/01/2010. 
Baretta, D., G. G. Brown \& E. J. B. Nogueira Cardoso. 2010. Potencial de la macrofauna y de otras variables edáficas como indicadoras de la calidad del suelo en áreas con Araucaria angustifolia. Acta Zoológica Mexicana (n.s.), Número Especial 2: 135-150.

RESUMEN. El estudio de la macrofauna del suelo en áreas con Araucaria angustifolia es importante para entender los procesos edáficos que ocurren en estos ecosistemas, ya que estos animales actúan en la descomposición y mineralización de la materia orgánica y en la manutención de la estructura del suelo. El objetivo del presente estudio fue evaluar, en áreas con araucaria naturales y reforestadas, impactadas o no por fuego accidental, el potencial indicador de la macrofauna edáfica y de variables ambientales (químicas y microbiológicas del suelo) para separar las áreas por medio del análisis canónico discriminante (ACD). Las áreas estudiadas incluyeron: bosque nativo con araucária, reforestación de araucaria, reforestación de araucaria sometida a fuego accidental y campo nativo con araucarias nativas y ocurrencia de fuego. En cada área, diez muestras de suelo fueron colectadas, próximo a los diez árboles de araucaria seleccionados al azar, en tres épocas diferentes, utilizando el método de excavación y colecta manual de monólitos (TSBF). Un impacto importante de la intervención antrópica fue observado sobre las propiedades biológicas y químicas del suelo. La macrofauna edáfica presentó potencial para ser usada como indicadora de la calidad del suelo. Los grupos Diplopoda, Chilopoda, Isoptera y Araneae y biomasa de la macrofauna, índice de diversidad de Shannon $(\mathrm{H})$, materia seca total de la hojarasca, fósforo y características microbiológicas, especialmente carbono de la biomasa microbiana y respiración basal fueron los responsables por casi toda la separación entre las áreas, siendo buenos indicadores de las modificaciones que ocurrieron en los ecosistemas. La contribución de cada variable para separar las áreas tuvo efecto estacional.

Palabras clave: Biodiversidad, análisis multivariado, análisis canónico discriminante, impactos ambientales, fuego accidental, lombrices de tierra.

\section{INTRODUÇÃO}

A Floresta Ombrófila Mista (Floresta de Araucária) raramente é encontrada em maciços extensos no Estado de São Paulo, sendo os remanescentes atuais, em sua maioria, secundários. No último inventário florestal realizado, verificou-se que as Florestas de Araucária cobrem apenas 174.681 ha dos solos paulistas (Kronka et al. 2005), grande parte concentrada na região de Campos do Jordão, SP (Baretta et al. 2007a). Este cenário de degradação das Florestas de Araucária ocorreu pela elevada importância sócio-econômica e ambiental da madeira e das sementes (pinhão) dessa espécie.

$\mathrm{O}$ estudo das comunidades de macrofauna e de outros atributos edáficos em áreas com araucária sob diferentes níveis de conservação pode ser um ponto de partida importante para entender os processos ocorrentes nos solos deste hábitat, já que estes organismos ocupam diversos níveis tróficos dentro da cadeia alimentar do solo. A macrofauna do solo desempenha uma variedade de funções nos ecossistemas, incluindo: decomposição da matéria orgânica, mineralização dos nutrientes, revolvimento, agregação do solo, proteção da planta contra pragas (controle biológico), e recuperação de áreas degradadas e contaminadas (Brown \& Fragoso 2003, Lavelle et al. 2006). Além disso, alguns grupos da macrofauna do solo têm sido amplamente usados como bioindicadores de distúrbios, bem como da qualidade do solo (Paoletti \& Bressan 1996, Paoletti 1999, Baretta et al. 2007b). Entretanto, os fatores responsáveis pelo 
desequilíbrio das comunidades de invertebrados edáficos ainda não estão elucidados e, atualmente, estão sendo investigados por poucos pesquisadores brasileiros.

Em trabalhos de campo, realizados para estudos de natureza ecológica, o grande desafio é desenvolver métodos para avaliar o impacto antropogênico sobre os processos biológicos nos quais os organismos participam. Qualquer indicador de qualidade do solo deve incluir, além das variáveis convencionais (físicas e químicas), atributos biológicos (como os microbiológicos e a macrofauna) do solo para que, em conjunto, possam refletir os processos que atuam na qualidade do solo. Como esses processos devem ser analisados de forma conjunta, torna-se necessária uma nova filosofia de análise para atender a essa demanda tão complexa, incorporando-se, nos estudos biológicos, a ferramenta de análise estatística multivariada (Baretta et al. 2006, Lima et al. 2007).

Vários estudos de biologia do solo no Brasil, especialmente sobre fauna edáfica tem utilizado técnicas de análise multivariada (Moço et al. 2005, Baretta et al. 2006, Baretta et al. 2007b), buscando maior entendimento das relações entre os invertebrados edáficos, além de identificar quais atributos biológicos do solo contribuem mais para discriminar os sistemas de manejo (Baretta et al. 2005, Baretta et al. 2006).

A hipótese deste estudo foi que o aumento da intervenção antrópica em áreas com araucária causa mais perdas do que ganhos na qualidade química e biológica do solo, reduzindo a diversidade da comunidade da macrofauna do solo. Desenvolveu-se o presente estudo com o objetivo de avaliar, em áreas com araucária naturais e reflorestadas, impactadas ou não pela queima acidental, o potencial indicador dos atributos da macrofauna edáfica e das variáveis ambientais (químicas e microbiológicas do solo) para separar as áreas e como indicadores da qualidade do solo, por meio de técnicas de análise multivariada.

\section{Local de estudo}

\section{MATERIAL E MÉTODOS}

O estudo foi realizado no município de Campos do Jordão, SP, em quatro áreas com Araucaria angustifolia, localizadas dentro do Parque Estadual de Campos do Jordão (PECJ), distante $210 \mathrm{~km}$ da cidade de São Paulo (22 ${ }^{\circ} 39^{\prime}$ latitude Sul e $45^{\circ} 27^{\prime}$ longitude Oeste), com altitude média de $1519 \mathrm{~m}$ (Baretta et al. 2008). O PECJ encontra-se em sua totalidade no Planalto de Campos do Jordão, com uma extensão de 8341,86 ha.

O clima da região é definido como subtropical de altitude, mesotérmico e úmido ( $\mathrm{Cfb}$, segundo a classificação de Köppen). A maior precipitação pluviométrica concentra-se no verão (dezembro a fevereiro), sendo que no mês de janeiro de 2005 (Época Chuvosa) ocorreram mais de $230 \mathrm{~mm}$, enquanto em agosto (2004/2005) (Época Seca) a escassez de chuvas foi acentuada, ocorrendo menos que $10 \mathrm{~mm}$ (Baretta et al. 2008). Em anos normais a precipitação anual é de $2000 \mathrm{~mm}$, com sazonalidade marcante, sendo o período seco de abril a setembro e o período chuvoso de outubro 
a março, quando ocorre $70 \%$ da precipitação anual (Baretta et al. 2008). O mês mais frio é julho, com temperatura média mensal próxima a $11,5^{\circ} \mathrm{C}$ e os meses mais quentes janeiro e fevereiro, com temperatura média superior a $17,0{ }^{\circ} \mathrm{C}$. Normalmente, podem ocorrer temperaturas abaixo de zero no inverno (Baretta et al. 2007b).

As áreas selecionadas incluíram vários estados de conservação das araucárias, sendo: 1) floresta nativa com predominância de araucária, em clímax ( $22^{\circ} 41^{\prime} 29^{\prime}$ " latitude Sul e 45 27' 52" longitude Oeste), com baixa interferência antrópica (NF); 2) reflorestamento de araucária ( $22^{\circ} 39^{\prime} 31^{\prime \prime}$ latitude Sul e $45^{\circ} 26^{\prime} 34$ " longitude Oeste), plantado em 1959 (R); 3) reflorestamento de araucária plantado em 1959 (22 39' 49”' latitude Sul e $45^{\circ} 26^{\prime} 58^{\prime \prime}$ longitude Oeste), submetido a um incêndio acidental intenso em julho de 2001 (RF) e 4) pastagem natural com araucárias nativas $\left(22^{\circ} 40^{\prime} 38^{\prime \prime}\right.$ latitude Sul e $45^{\circ} 28^{\prime} 18^{\prime \prime}$ longitude Oeste), submetida a incêndio acidental intenso em setembro de 2004 (NPF). Todos os locais de amostra apresentam topo-seqüências e altitudes semelhantes. O relevo é suave ondulado, com declividade média das áreas de $0,1702 \mathrm{~m} \mathrm{~m}^{-1}$. O solo nas áreas com araucária foi classificado como Latossolo Vermelho-Amarelo distrófico. Maiores informações sobre as características químicas do solo podem ser obtidas em Baretta et al. (2007b).

A floresta nativa (NF) é composta basicamente por Araucaria angustifolia (Bertoloni) Otto Kuntze e por diversas espécies arbustivas, herbáceas e arbóreas pertencentes às famílias Aquifoliaceae, Araucariaceae, Asteraceae, Cyatheaceae, Lauraceae, Myrsinaceae, Myrtaceae, Podocarpaceae, Rhamnaceae, Rosaceae, Simaroubaceae, Solanaceae, Symplocaceae, Vochysiaceae e Winteraceae. A área R apresenta maior densidade de araucárias e várias árvores de Podocarpus lambertii Klotz (Podocarpaceae), das mesmas espécies da área NF. A área RF apresenta menor densidade de araucária e um predomínio de gramíneas Aristida longiseta (Poaceae) e da composta Braccharis trimera (Compositae). É comum observar a presença de bovinos pastejando nos reflorestamentos R e RF, e na área NPF, em baixas lotações. Maiores informações sobre a flora arbórea, arbustiva e herbácea do Parque Estadual de Campos do Jordão podem ser obtidas em Baretta et al. (2007b).

\section{Avaliação dos atributos biológicos e químicos do solo}

As coletas da comunidade de macrofauna invertebrada do solo foram realizadas em setembro de 2004 (período seco; Época 1 = E1), fevereiro de 2005 (período chuvoso; Época 2 = E2) e agosto de 2005 (período seco; Época $3=$ E3). A caracterização do "período seco" e "período úmido" levou em conta a média de precipitação dos 4 meses anteriores às coletas. Em cada área, foram escolhidas, ao acaso, dez árvores de araucária e os pontos de amostragem da macrofauna distribuídos perto de cada árvore selecionada, sob a copa de cada uma. O método de amostragem foi padronizado para todos os atributos edáficos, visando sua utilização na análise multivariada. 
A avaliação da comunidade de macrofauna invertebrada do solo foi realizada por meio do método recomendado pelo Programa "Tropical Soil Biology and Fertility" (TSBF) descrito por Anderson \& Ingram (1993). Dez monólitos de solo de $25 \times 25$ $\mathrm{cm}$ de lado e $30 \mathrm{~cm}$ de profundidade foram coletados, ao acaso, perto de cada árvore de araucária selecionada. Antes da retirada do solo a serapilheira foi amostrada na mesma área do monólito. A porcentagem de água contida nas amostras da serapilheira foi determinada, obtendo-se a quantidade de matéria seca total (MST). Os pontos de amostragem apresentaram distância mínima de 10 metros entre si.

Os organismos da macrofauna do solo visíveis a olho nú, foram separados manualmente da serapilheira e do solo, e identificados em nível de ordem ou espécie (no caso de minhocas, veja Baretta et al. 2007b), com auxílio de microscópio estereoscópico com aumento de $100 \times$. Em seguida, foram reunidos novamente e realizada a pesagem de todos os organismos, após 15 minutos de secagem ao ar livre sobre papel absorvente, obtendo-se a biomassa fresca total ( $\mathrm{g}$ de peso fresco por $\mathrm{m}^{2}$ ). Depois, os indivíduos foram etiquetados, colocados em frascos apropriados e conservados em álcool etílico a $75 \%$. Parte do material coletado, especialmente os artrópodes estão depositados na coleção de Aracnídeos do Laboratório de Artrópodes do Instituto Butantan (A.D. Brescovit, curador) (Baretta et al. 2007a).

Para a avaliação dos atributos químicos e microbiológicos do solo foram realizadas coletas nas mesmas épocas e pontos de amostragem da macrofauna (setembro de 2004, fevereiro de 2005 e agosto de 2005). Para tanto, sob a copa (no mínimo a $1 \mathrm{~m}$ e no máximo a $2 \mathrm{~m}$ de distância de cada árvore) das mesmas árvores escolhidas para a coleta da macrofauna, foram retiradas amostras de solo na profundidade de 0-20 $\mathrm{cm}$. Para as análises, as amostras foram homogeneizadas e passadas em peneira com malha de $2 \mathrm{~mm}$. Uma subamostra foi retirada (somente em agosto de 2004), para determinação dos seguintes atributos químicos do solo: $\mathrm{pH} \mathrm{em} \mathrm{CaCl}_{2}$, matéria orgânica (Método Walkey-Black), teores de fósforo (Resina), potássio, cálcio e magnésio trocáveis, conforme metodologia descrita em Raij et al. (2001). O carbono da biomassa microbiana (CBM) foi determinado pelo método da fumigação-extração (Vance et al. 1987). O carbono orgânico total (COT) do solo foi estimado por oxidação úmida com dicromato de potássio $\left(\mathrm{K}_{2} \mathrm{Cr}_{2} \mathrm{O}_{4}\right)$ e ácido sulfúrico $\left(\mathrm{H}_{2} \mathrm{SO}_{4}\right)$, conforme proposto por Raij et al. (2001). A atividade microbiana foi avaliada pela determinação da respiração basal $\left(\mathrm{C}-\mathrm{CO}_{2}\right)$ em $50 \mathrm{~g}$ de amostra de solo (Alef \& Nannipieri 1995). Utilizaramse os resultados de C- $\mathrm{CO}_{2}$ e do CBM para calcular o quociente metabólico $\left(q \mathrm{CO}_{2}\right)$, conforme proposto por Anderson \& Domsch (1993).

\section{Análise estatística}

A partir do resultado do número de indivíduos por metro quadrado (densidade total), foi calculado o índice de diversidade de Shannon (H) (Odum 1983). A densidade relativa (Indiv. $\mathrm{m}^{-2}$ ) de cada grupo taxonômico da macrofauna do solo nas diferentes 
áreas foi utilizada para a obtenção do comprimento do gradiente. Como este comprimento foi menor que três (resposta linear), optou-se pela Análise de Componentes Principais (ACP), usando o programa CANOCO versão 4.0 (ter Braak \& Smilauer 1998). Os atributos químicos ( $\mathrm{pH}, \mathrm{MO}, \mathrm{P}, \mathrm{K}, \mathrm{Ca}, \mathrm{Mg}, \mathrm{H}+\mathrm{Al})$ e microbiológicos do solo (CBM, C- $\mathrm{CO}_{2}$, COT, relação CBM:COT e $q \mathrm{CO}_{2}$ ), MST e umidade do solo foram utilizados posteriormente na $\mathrm{ACP}$, como variáveis ambientais explicativas das modificações dos atributos da macrofauna [densidade de Oligochaeta, Formicidae, Coleoptera, Enchytraeidae, Araneae, Chilopoda, Diplopoda, Opilionidae, Mollusca, Isoptera, Pseudoescorpionida, outros grupos menos freqüentes, densidade total, $\mathrm{H} \mathrm{e}$ Biomassa da macrofauna] (ter Braak \& Smilauer 1998). Adicionalmente, as variáveis ambientais e os principais atributos da macrofauna foram submetidos à análise canônica discriminante (ACD) para identificar quais deles foram mais relevantes na separação das áreas amostradas (Cruz-Castillo et al. 1994, Baretta et al. 2005, Maluche-Baretta et al. 2006).

\section{RESULTADOS E DISCUSSÃO}

\section{Análise de Componentes Principais (ACP)}

A variabilidade dos dados nas três épocas de amostragem foi explicada em $22,5 \%$ pela componente principal 1 (CP 1) e 12,7\% pela componente principal 2 (CP 2), totalizando 35,2\% da variabilidade total dos dados da macrofauna analisados (Fig. 1). A terceira componente principal (CP 3) explicou, nas três épocas de amostragem (E1, E2 e E3), menos do que 10,0\% da variabilidade total e não foi apresentada.

A relação entre as CP 1 e CP 2 demonstrou que a área NF ficou separada das outras áreas nas três épocas de coleta e sofreu menos mudanças quanto a sua disposição no espaço temporal. Essa floresta nativa caracterizou-se por apresentar maiores valores de praticamente todos os atributos da macrofauna (Opilionidae, Chilopoda, Oligochaeta, Araneae, Diplopoda, índice de diversidade de Shannon) e das variáveis ambientais (CBM, C-CO ${ }_{2}$, relação CBM:COT, COT, P, Ca, $\mathrm{Mg}$, umidade do solo e MST).

\section{Análise Canônica Discriminante (ACD)}

O teste estatístico multivariado de Wilks' Lambda para os atributos da macrofauna e variáveis ambientais demonstrou haver diferenças significativas entre as épocas de amostragem e áreas estudadas $(\mathrm{p}<0,0001)$ quanto à função canônica discriminante $1\left(\mathrm{FCD}_{1}\right)$ e $2\left(\mathrm{FCD}_{2}\right)$, por isso realizou-se uma $\mathrm{ACD}$ para cada época de amostragem (Fig. 2). A relação CBM:COT e o carbono orgânico total (COT) do solo são relações de divisão direta e altamente influenciadas pelos valores de CBM e matéria orgânica (MO), por isso foram retiradas da ACD, aumentando a confiabilidade dessa análise, pela eliminação do efeito de colinearidade dos dados. 


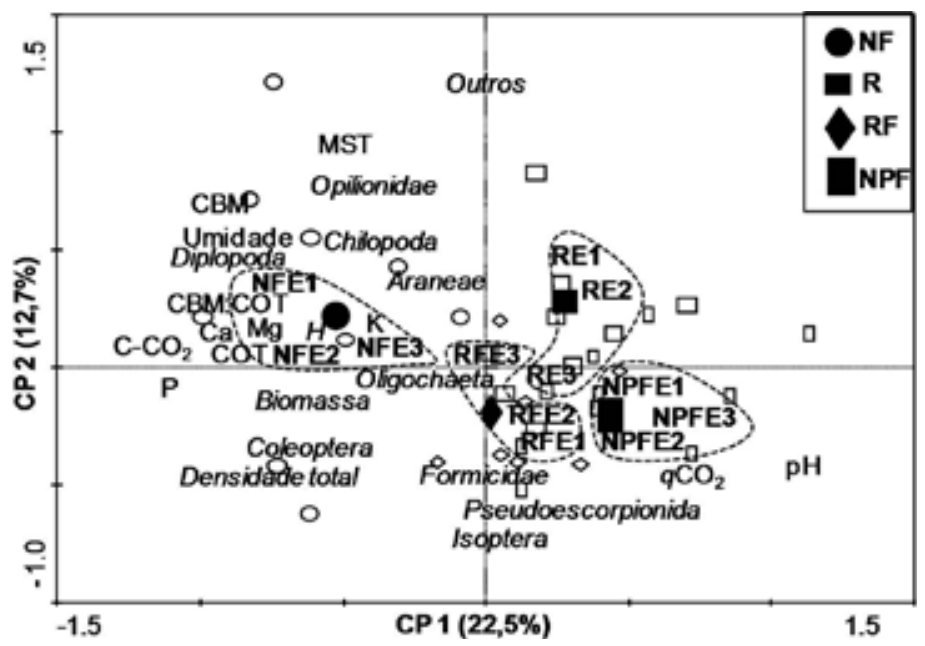

Figura 1. Relação entre as componentes principais 1 e 2 (CP 1 e CP 2) da análise de componentes principais (ACP), discriminando Florestas de Araucária nativa (NF), introduzida (R), introduzida impactada (RF) e área de pastagem natural com araucárias nativas impactada pela queima acidental

(NPF), atributos da macrofauna do solo [Em itálico na figura= são os grupos mais freqüentes da macrofauna do solo; Outros = somatório dos grupos de organismos menos freqüentes; Biomassa = biomassa fresca total da macrofauna e $\mathrm{H}=$ índice de diversidade de Shannon) e variáveis ambientais explicativas $[\mathrm{pH}=$ potencial hidrogeniônico do solo; $\mathrm{P}=$ fósforo; $\mathrm{K}=$ potássio; $\mathrm{Ca}=$ cálcio; $\mathrm{Mg}=$ magnésio; Umidade $=$ umidade do solo; $\mathrm{MST}=$ matéria seca total da serapilheira; $\mathrm{COT}=$ carbono orgânico total do solo; $\mathrm{C}-\mathrm{CO}_{2}=$ respiração basal do solo; $\mathrm{CBM}$ = carbono da biomassa microbiana; $q \mathrm{CO}_{2}=$ quociente metabólico e relação CBM:COT)] em setembro de 2004 (E1 = Época 1), fevereiro de 2005 (E2 = Época 2) e agosto de 2005 (E3 = Época 3). Símbolo cheio representa o valor médio das três épocas de coleta (centróide) para cada área $(\mathrm{n}=10)$.

O modelo estatístico utilizado na ACD explicou boa parte da variabilidade presente nas áreas, uma vez que a $\mathrm{FCD}_{1}$ e $\mathrm{FCD}_{2}$ apresentaram correlações canônicas de 64 e $28 \%$ na amostragem de setembro (2004), de 55 e 35\% em fevereiro (2005), e de 60 e 34\% em agosto (2005), respectivamente (Fig. 2). Essas duas funções foram ajustáveis para explicar as variações encontradas nos valores dos atributos ambientais e da macrofauna do solo. Os altos valores de correlação também indicaram elevada associação entre os atributos analisados e as áreas de coleta desses atributos.

$\mathrm{Na}$ Fig. 2, estão indicados os coeficientes canônicos padronizados (CCP) da $\mathrm{FCD}_{1}$ e $\mathrm{FCD}_{2}$, para as quatro áreas com araucária amostradas, considerando todos os atributos ambientais e da macrofauna analisados em cada época de amostragem. O CCP explica o comportamento multivariado dos diferentes atributos para promover a separação entre as áreas com araucária, em resposta ao estudo das variáveis independentes, analisadas simultaneamente (Hair et al. 1987, Cruz-Castillo et al. 1994, Baretta et al. 2006). 

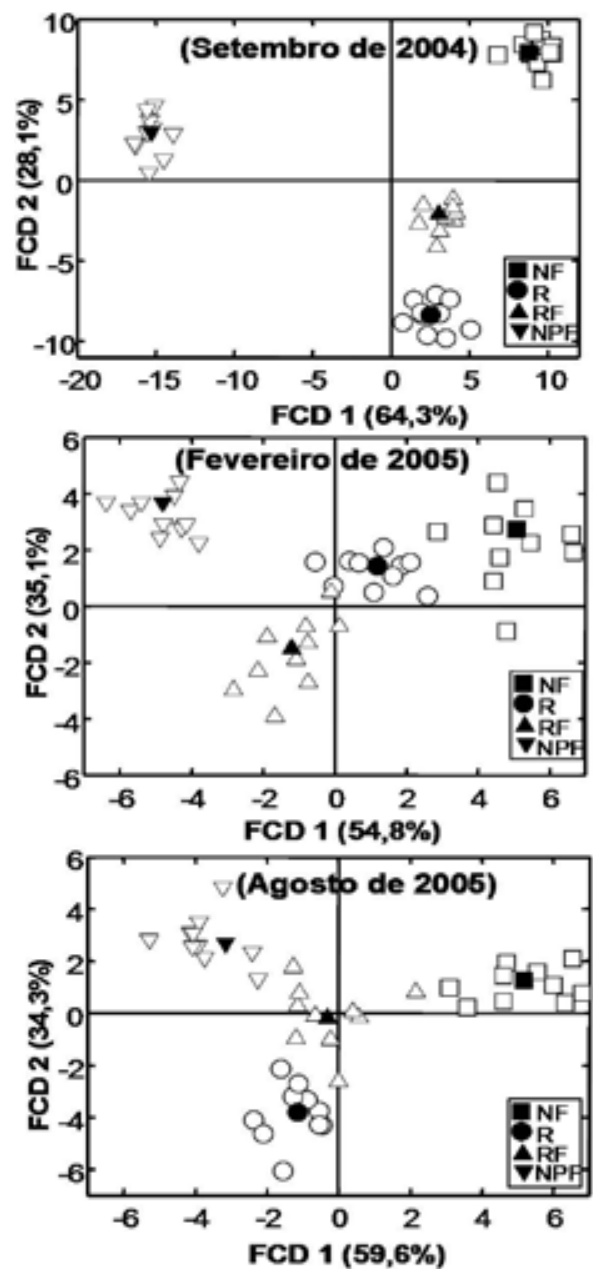

Figura 2. Coeficientes canônicos padronizados $(\mathrm{CCP})$ da função canônica discriminante 1 e $2\left(\mathrm{FCD}_{1}\right.$ e $\mathrm{FCD}_{2}$ ), discriminando Florestas de Araucária nativa (NF), introduzida (R), introduzida impactada

(RF) e área de pastagem natural com araucárias nativas impactada pela queima acidental (NPF), considerando os atributos das variáveis ambientais e da macrofauna do solo coletados com o método TSBF $(25 \times 25 \mathrm{~cm})$, em setembro de 2004 (E1 = Época 1), fevereiro de 2005 (E2 = Época 2) e agosto de 2005 (E3 = Época 3), na região de Campos do Jordão, SP. Símbolo cheio representa o valor médio de CCP (centróide) para cada área $(\mathrm{n}=10)$.

De maneira geral, observou-se nas três épocas de amostragem, que a $\mathrm{FCD}_{1}$ separou a área $\mathrm{NF}$, com maiores valores de $\mathrm{CCP}$, das outras áreas, especialmente da área NPF, que ficou afastada das demais. As áreas R e RF ficaram numa posição intermediária e foram muito similares entre si, independente da época de amostragem, 
TABELA I. Coeficiente de correlação canônica $(r)$, coeficiente canônico padronizado (CCP) e coeficiente da taxa de discriminação paralela (TDP) quanto às funções canônicas discriminantes 1 e $2\left(\mathrm{FCD}_{1}\right.$ e $\left.\mathrm{FCD}_{2}\right)$, referente aos atributos das variáveis ambientais e da macrofauna do solo, independente do tratamento, na região de Campos do Jordão, SP, em Setembro de $2004(n=40)$.

\begin{tabular}{|c|c|c|c|c|c|c|}
\hline \multirow[b]{2}{*}{ Variáveis ambientais } & \multicolumn{3}{|c|}{$\mathrm{FCD}_{1}(64,3 \%)$} & \multicolumn{3}{|c|}{$\mathrm{FCD}_{2}(28,1 \%)$} \\
\hline & $r$ & $\mathrm{CCP}$ & TDP & $r$ & $\mathrm{CCP}$ & TDP \\
\hline Carbono da Biomassa Microbiana (CBM) & 0,17 & 0,44 & 0,07 & 0,05 & $-0,54$ & $-0,03$ \\
\hline Respiração basal do solo $\left(\mathrm{C}-\mathrm{CO}_{2}\right)$ & 0,13 & 0,52 & 0,07 & 0,06 & 0,62 & 0,04 \\
\hline Quociente metabólico $\left(q \mathrm{CO}_{2}\right)$ & $-0,13$ & 0,35 & $-0,05$ & 0,04 & $-1,60$ & $-0,06$ \\
\hline $\mathrm{pH}$ do solo $(\mathrm{pH})^{1}$ & $-0,07$ & $-0,06$ & 0,00 & $-0,11$ & 1,35 & $-0,15$ \\
\hline Matéria Orgânica $(\mathrm{MO})^{1}$ & 0,12 & 0,58 & 0,07 & $-0,06$ & $-5,18$ & 0,31 \\
\hline Fósforo $(\mathrm{P})^{1}$ & 0,13 & 0,83 & 0,11 & 0,12 & 1,11 & 0,13 \\
\hline Potássio $(\mathrm{K})^{1}$ & $-0,00$ & $-0,45$ & 0,00 & 0,08 & $-1,08$ & $-0,09$ \\
\hline Cálcio $(\mathrm{Ca})^{1}$ & $-0,03$ & $-0,12$ & 0,00 & 0,13 & 3,25 & 0,42 \\
\hline Magnésio $(\mathrm{Mg})^{1}$ & 0,02 & $-0,83$ & $-0,02$ & 0,10 & 0,35 & 0,04 \\
\hline Hidrogênio + Alumínio $(\mathrm{H}+\mathrm{Al})^{1}$ & 0,09 & 0,23 & 0,02 & $-0,03$ & 2,89 & $-0,09$ \\
\hline Umidade do solo & 0,12 & 0,87 & 0,10 & $-0,03$ & $-0,14$ & 0,00 \\
\hline \multicolumn{7}{|l|}{ Grupos mas freqüentes da macrofauna } \\
\hline Oligochaeta & 0,06 & 0,05 & 0,00 & 0,04 & $-0,54$ & $-0,02$ \\
\hline Formicidae & $-0,01$ & $-0,12$ & 0,00 & 0,06 & 1,09 & 0,07 \\
\hline Isoptera & $-0,01$ & 0,36 & 0,00 & $-0,01$ & $-0,77$ & 0,01 \\
\hline Coleoptera & $-0,03$ & $-0,28$ & 0,01 & 0,06 & $-2,22$ & $-0,13$ \\
\hline Araneae & 0,06 & 0,44 & 0,03 & 0,07 & 0,34 & 0,02 \\
\hline Chilopoda & 0,06 & 1,35 & 0,08 & 0,05 & 0,79 & 0,04 \\
\hline Diplopoda & 0,05 & 0,18 & 0,01 & 0,12 & 0,36 & 0,04 \\
\hline Opilionidae & 0,02 & $-0,04$ & 0,00 & 0,05 & 2,43 & 0,12 \\
\hline Mollusca & 0,04 & 1,00 & 0,05 & 0,07 & 0,69 & 0,05 \\
\hline Pseudoescorpionida & $-0,02$ & 0,16 & 0,00 & $-0,02$ & $-0,73$ & 0,01 \\
\hline Outros $^{2}$ & 0,01 & $-0,18$ & 0,00 & $-0,01$ & $-0,51$ & 0,01 \\
\hline \multicolumn{7}{|l|}{ Outros atributos da macrofauna } \\
\hline Densidade relativa $\left(\right.$ Ind. $\mathrm{m}^{-2}$ ) & 0,06 & 0,10 & 0,00 & 0,02 & 1,57 & 0,03 \\
\hline Índice de Diversidade de Shannon $(\mathrm{H})$ & 0,07 & $-0,33$ & $-0,02$ & 0,04 & 2,58 & 0,10 \\
\hline Biomassa fresca total da macrofauna $\left(\mathrm{g} \mathrm{m}^{2}\right)$ & 0,09 & 0,97 & 0,09 & 0,07 & $-0,29$ & $-0,02$ \\
\hline
\end{tabular}

${ }^{1}$ Parâmetros químicos do solo avaliados somente na primeira época (setembro de 2004); ${ }^{2}$ Outros = somatório dos grupos de organismos menos freqüentes.

em termos de atributos ambientais e da macrofauna do solo. A diferença em termos de desvios dos valores de CCP em relação ao centróide aumenta com o tempo após a queima acidental, de setembro de 2004 para agosto de 2005. A separação entre as áreas amostradas torna-se mais nítida em setembro (2004), logo após o impacto da 
TABELA II. Coeficiente de correlação canônica $(r)$, coeficiente canônico padronizado (CCP) e coeficiente da taxa de discriminação paralela (TDP) quanto às funções canônicas discriminantes 1 e $2\left(\mathrm{FCD}_{1}\right.$ e $\left.\mathrm{FCD}_{2}\right)$, referente aos atributos das variáveis ambientais e da macrofauna do solo, independente do tratamento, na região de Campos do Jordão, SP, em Fevereiro de $2005(n=40)$.

\begin{tabular}{|c|c|c|c|c|c|c|}
\hline \multirow[b]{2}{*}{ Variáveis ambientais } & \multicolumn{3}{|c|}{$\mathrm{FCD}_{1}(54,8 \%)$} & \multicolumn{3}{|c|}{$\mathrm{FCD}_{2}(35,1 \%)$} \\
\hline & $r$ & $\mathrm{CCP}$ & TDP & $r$ & $\mathrm{CCP}$ & TDP \\
\hline Carbono da Biomassa Microbiana (CBM) & 0,20 & $-0,12$ & $-0,02$ & 0,22 & 0,30 & 0,07 \\
\hline Respiração basal do solo $\left(\mathrm{C}-\mathrm{CO}_{2}\right)$ & 0,13 & 1,33 & 0,17 & $-0,07$ & $-0,86$ & 0,06 \\
\hline Quociente metabólico $\left(q \mathrm{CO}_{2}\right)$ & $-0,09$ & $-1,58$ & 0,14 & 0,19 & 0,81 & 0,15 \\
\hline Umidade do solo & 0,26 & $-0,17$ & $-0,04$ & 0,22 & 0,40 & 0,09 \\
\hline Matéria seca da serapilheira (MST) & 0,37 & 0,82 & 0,30 & 0,15 & 0,14 & 0,02 \\
\hline \multicolumn{7}{|l|}{ Grupos mas freqüentes da macrofauna } \\
\hline Oligochaeta & 0,03 & 0,11 & 0,00 & $-0,15$ & $-0,21$ & 0,03 \\
\hline Formicidae & $-0,07$ & 0,31 & $-0,02$ & 0,06 & $-0,74$ & $-0,04$ \\
\hline Isoptera & $-0,06$ & $-1,12$ & 0,07 & 0,13 & 0,97 & 0,13 \\
\hline Coleoptera & 0,05 & $-0,56$ & $-0,03$ & 0,06 & $-1,26$ & $-0,08$ \\
\hline Araneae & 0,07 & $-0,27$ & $-0,02$ & 0,18 & 0,35 & 0,06 \\
\hline Chilopoda & 0,18 & 0,69 & 0,12 & 0,04 & $-0,08$ & $-0,00$ \\
\hline Diplopoda & 0,30 & 1,07 & 0,32 & 0,15 & 0,77 & 0,12 \\
\hline Opilionidae & 0,13 & $-0,33$ & $-0,04$ & 0,08 & 0,36 & 0,03 \\
\hline Mollusca & 0,00 & 0,00 & 0,00 & 0,00 & 0,00 & 0,00 \\
\hline Pseudoescorpionida & 0,00 & 0,00 & 0,00 & 0,00 & 0,00 & 0,00 \\
\hline Outros $^{1}$ & 0,11 & 0,67 & 0,07 & 0,18 & 0,42 & 0,08 \\
\hline \multicolumn{7}{|l|}{ Outros atributos da macrofauna } \\
\hline Densidade relativa (Ind. $\mathrm{m}^{-2}$ ) & 0,05 & 1,31 & 0,07 & 0,15 & 1,89 & 0,28 \\
\hline Índice de Diversidade de Shannon (H) & 0,17 & $-0,26$ & $-0,04$ & 0,17 & 0,16 & 0,03 \\
\hline Biomassa fresca total da macrofauna $\left(\mathrm{g} \mathrm{m}^{2}\right)$ & 0,07 & $-0,41$ & $-0,03$ & 0,02 & 0,11 & 0,00 \\
\hline
\end{tabular}

${ }^{1}$ Outros $=$ somatório dos grupos de organismos menos freqüentes.

queima na área NPF, em comparação às outras épocas de amostragem (fevereiro e agosto de 2005) (Fig. 2).

\section{Coeficiente da taxa de discriminação paralela (TDP)}

$\mathrm{O}$ valor de TDP resulta do produto entre os coeficientes canônicos padronizados $(\mathrm{CCP})$ e de correlação $(r)$. O $r$ mostra informações univariadas (contribuição individual) de cada atributo, independente dos demais. Contudo, o melhor parâmetro para avaliação do efeito de separação gerada pelos atributos dentro das áreas é o TDP. Quanto ao coeficiente TDP, valores positivos indicam efeito de separação entre as áreas, enquanto valores negativos semelhanças entre as áreas quanto a esse atributo (Baretta et al. 2006). 
TABELA III. Coeficiente de correlação canônica (r), coeficiente canônico padronizado (CCP) e coeficiente da taxa de discriminação paralela (TDP) quanto às funções canônicas discriminantes 1 e $2\left(\mathrm{FCD}_{1}\right.$ e $\left.\mathrm{FCD}_{2}\right)$, referente aos atributos das variáveis ambientais e da macrofauna do solo, independente do tratamento, na região de Campos do Jordão, SP, em Agosto de $2005(n=40)$.

\begin{tabular}{|c|c|c|c|c|c|c|}
\hline & \multicolumn{3}{|c|}{$\mathrm{FCD}_{1}(59,6 \%)$} & \multicolumn{3}{|c|}{$\mathrm{FCD}_{2}(34,3 \%)$} \\
\hline & $r$ & CCP & TDP & $r$ & $\mathrm{CCP}$ & TDP \\
\hline \multicolumn{7}{|l|}{ Variáveis ambientais } \\
\hline Carbono da Biomassa Microbiana (CBM) & 0,22 & 0,61 & $-0,13$ & $-0,23$ & $-0,33$ & 0,08 \\
\hline Respiração basal do solo $\left(\mathrm{C}-\mathrm{CO}_{2}\right)$ & 0,12 & $-0,38$ & $-0,05$ & $-0,19$ & $-2,17$ & 0,41 \\
\hline Quociente metabólico $\left(q \mathrm{CO}_{2}\right)$ & $-0,16$ & $-1,97$ & 0,32 & 0,20 & 0,67 & 0,13 \\
\hline Umidade do solo & $-0,10$ & 0,86 & $-0,09$ & 0,13 & $-0,47$ & $-0,06$ \\
\hline $\begin{array}{l}\text { Matéria seca total da serapilheira (MST) } \\
\text { Grupos mas freqüentes da macrofauna }\end{array}$ & 0,35 & 0,75 & 0,26 & $-0,31$ & $-1,12$ & 0,35 \\
\hline Oligochaeta & $-0,04$ & 0,29 & 0,01 & 0,07 & $-0,01$ & 0,00 \\
\hline Formicidae & 0,09 & $-0,23$ & $-0,02$ & 0,06 & 0,39 & 0,02 \\
\hline Isoptera & $-0,08$ & $-1,09$ & 0,09 & 0,05 & 0,34 & 0,02 \\
\hline Coleoptera & 0,14 & 0,26 & 0,04 & 0,18 & 1,15 & 0,21 \\
\hline Araneae & 0,04 & $-0,33$ & $-0,01$ & 0,12 & $-0,10$ & $-0,01$ \\
\hline Chilopoda & 0,18 & 0,35 & 0,06 & $-0,11$ & 0,08 & $-0,01$ \\
\hline Diplopoda & 0,38 & 1,46 & 0,55 & 0,14 & 0,59 & 0,08 \\
\hline Opilionidae & 0,00 & 0,00 & 0,00 & 0,00 & 0,00 & 0,00 \\
\hline Mollusca & 0,00 & 0,00 & 0,00 & 0,00 & 0,00 & 0,00 \\
\hline Pseudoescorpionida & $-0,05$ & 0,25 & $-0,01$ & 0,05 & $-0,94$ & $-0,05$ \\
\hline Outros $^{1}$ & $-0,08$ & 0,78 & $-0,06$ & 0,12 & $-1,59$ & $-0,19$ \\
\hline \multicolumn{7}{|l|}{ Outros atributos da macrofauna } \\
\hline Densidade relativa $\left(\right.$ Ind. $\mathrm{m}^{-2}$ ) & 0,13 & 0,85 & 0,11 & 0,09 & 0,43 & 0,04 \\
\hline Índice de Diversidade de Shannon $(\mathrm{H})$ & 0,23 & $-0,49$ & $-0,11$ & $-0,06$ & 0,66 & $-0,04$ \\
\hline Biomassa fresca total da macrofauna $\left(\mathrm{g} \mathrm{m}^{2}\right)$ & 0,04 & 0,21 & 0,01 & 0,02 & $-0,12$ & 0,00 \\
\hline
\end{tabular}

${ }^{1}$ Outros $=$ somatório dos grupos de organismos menos freqüentes.

De maneira geral, o valor de TDP de cada atributo foi diferenciado para cada época de amostragem. Na amostragem de setembro (2004), dentro da $\mathrm{FCD}_{1}$, os atributos MST $(0,44), \mathrm{P}(0,11)$, umidade do Solo $(0,10)$, MO $(0,07)$, CBM $(0,07)$ e C-CO $(0,07)$ apresentaram os maiores valores positivos de coeficientes TDP, indicando que o maior efeito de separação das áreas é explicado, principalmente, por estas variáveis ambientais (Tabela I). $\mathrm{Na} \mathrm{FCD}_{2}$, as variáveis ambientais com maior valor de TDP foram $\mathrm{Ca}(0,42), \mathrm{MO}(0,31), \mathrm{P}(0,13)$ e MST $(0,11)$. Os atributos da macrofauna considerados indicadores de mudanças nas áreas na $\mathrm{FCD}_{1}$ para a primeira coleta (setembro de 2004) foram biomassa da macrofauna $(0,09)$ e os grupos Chilopoda $(0,08)$ e Mollusca $(0,05)$. $\mathrm{Na} \mathrm{FCD}_{2}$, os grupos da macrofauna com maior valor de TDP foram 
Opilionidae $(0,12)$, índice de diversidade de Shannon $(0,10)$ e Formicidae $(0,07)$, respectivamente. Os demais atributos das variáveis ambientais e da macrofauna foram menos sensíveis, com menores valores de TDP e contribuíram menos na função indicada para a separação entre as áreas (Tabela I).

$\mathrm{Na}$ amostragem de fevereiro (2005), dentro da $\mathrm{FCD}_{1}$, os atributos MST $(0,30)$, C$\mathrm{CO}_{2}(0,17)$ e $q \mathrm{CO}_{2}(0,14)$ apresentaram os maiores valores positivos de coeficientes TDP (Tabela II). Os atributos da macrofauna que apresentaram maiores valores de TDP na $\mathrm{FCD}_{1}$ foram os grupos Diplopoda $(0,32)$, Chilopoda $(0,12)$, Isoptera $(0,07)$ e a densidade relativa da macrofauna $(0,07) . \mathrm{Na} \mathrm{FCD}_{2}$, os atributos $q \mathrm{CO}_{2}(0,15)$, umidade do solo $(0,09)$ e CBM $(0,07)$ apresentaram os maiores valores positivos de coeficientes TDP. Os atributos da macrofauna que apresentaram maiores valores de TDP na $\mathrm{FCD}_{2}$ foram densidade relativa da macrofauna $(0,28)$ e os grupos Isoptera $(0,13)$, Diplopoda $(0,12)$ e Outros $(0,08)$. Portanto, em fevereiro $(2005)$ de todos os atributos das variáveis ambientais e da macrofauna estudados, o grupo Diplopoda foi o que contribuiu mais para a separação entre as áreas, sendo um bom indicador das interferências antrópicas.

$\mathrm{Na}$ amostragem de agosto (2005), dentro da $\mathrm{FCD}_{1}$, os atributos $q \mathrm{CO}_{2}(0,32) \mathrm{e}$ MST $(0,26)$, e na $\mathrm{FCD}_{2}$, os atributos $\mathrm{C}-\mathrm{CO}_{2}(0,41)$ e MST $(0,35)$, apresentaram os maiores valores positivos de coeficientes TDP (Tabela III). Os atributos da macrofauna com maiores valores de TDP na $\mathrm{FCD}_{1}$ foram os grupos Diplopoda $(0,55)$, Isoptera $(0,09)$ e a densidade relativa da macrofauna $(0,11)$, enquanto na $\mathrm{FCD}_{2}$, foram os grupos Coleoptera $(0,21)$ e Diplopoda $(0,08)$.

\section{DISCUSSÃO}

No presente estudo, a NF apresentou maior diversidade e abundância da macrofauna do solo (Opilionidae, Chilopoda, Oligochaeta, Araneae, Diplopoda) e melhores condições ambientais (CBM, C- $\mathrm{CO}_{2}$, relação CBM:COT, COT, $\mathrm{P}, \mathrm{Ca}, \mathrm{Mg}$, umidade do solo e MST, menor condição de estresse $=$ menor $q \mathrm{CO}_{2}$ ) do que nos demais tratamentos avaliados. A maior riqueza de grupos, especialmente dos predadores (Chilopoda, Opilionidae, Araneae, etc) foi acompanhada de uma melhoria da qualidade do solo (Paoletti 1999), e permite uma primeira inferência sobre o grau de complexidade e das interações ecológicas existentes entre as comunidades de macroinvertebrados (Stork \& Eggleton 1992) e as propriedades do solo.

Outro fator que poderia estar contribuindo para a melhor qualidade química e biológica do solo da área NF refere-se à quantidade da serapilheira (MST) e do material orgânico, que é determinada, entre outros aspectos, pela diversidade florística de cada área (Baretta et al. 2008). A manutenção de uma cobertura florística mais diversa na superfície do solo aumenta a umidade e diminui as oscilações térmicas (Cochran et al. 1994). Contudo, a diversidade da macrofauna do solo depende de muitos outros fatores, como tipo de manejo, disponibilidade de fontes de alimento, teor de matéria 
orgânica e umidade do solo (Kladivko 2001), ficando os invertebrados mais suscetíveis às mudanças microclimáticas do hábitat.

Diversos estudos mostram que a comunidade de macrofauna do solo pode diminuir em abundância e riqueza de grupos taxonômicos na estação seca após o fogo (Athias et al. 1975, Bandeira \& Harada 1998, Baretta et al. 2005, Baretta et al. 2007a). No presente caso, as condições adversas, como o fogo acidental, diminuição da cobertura vegetal, oscilações de temperatura e umidade do solo, criaram uma condição de estresse na área NPF, com diminuição das características químicas e dos atributos biológicos do solo, incluindo redução da riqueza de grupos funcionais da macrofauna edáfica. Constatou-se também que a área NPF, ao contrário da NF, caracterizou-se por apresentar nas três épocas de amostragem (E1, E2 e E3), maiores valores de pH e de $q \mathrm{CO}_{2}$ e, por conseqüência, maiores perdas de $\mathrm{C}$.

Estudos recentes mostram que o TDP é mais eficiente quando o pesquisador deseja discriminar áreas (Cruz-Castillo et al. 1994, Baretta et al. 2005, Baretta et al. 2006, Maluche-Baretta et al. 2006). Portanto, a ACD apresenta muitas vantagens em relação à $\mathrm{ACP}$, pois têm um teste estatístico multivariado (Wilks' Lambda) que indica se as áreas estudadas são diferentes entre si, além de fornecer a contribuição real de cada atributo edáfico para esta separação (Baretta et al. 2006, Maluche-Baretta et al. 2006). Valores positivos de TDP indicam efeito de separação entre as áreas, enquanto valores negativos semelhanças entre as áreas quanto a esse atributo (Baretta et al. 2006). No presente estudo, observou-se através dos valores de TDP, que diversos atributos ambientais e da macrofauna do solo foram eficientes para separar as áreas amostradas, apresentando certo potencial (maior valor de TDP) como indicadores (Tabelas I, II, III), independente da época de amostragem, pois promoveram uma boa separação entre as áreas (Fig. 2).

A aplicação do TDP permitiu obter um valor indicador para os atributos estudados. A partir desses resultados de TDP da análise ACD propõe-se a separação dos indicadores em seis classes, de acordo com seu valor de indicador (Tabela IV). Isso pode auxiliar na escolha dos atributos do solo que são mais sensíveis para promover diferenças entre os tratamentos, e evitar a perda de tempo e dinheiro com variáveis edáficas que não respondem aos efeitos testados.

Como não foi um atributo edáfico isoladamente que apresentou potencial para separar as áreas, nenhuma medida isolada pode fornecer uma ampla visão da qualidade do solo. É necessário sempre realizar uma avaliação integrada dos diversos atributos dessa qualidade (Lima et al. 2007, Baretta et al. 2008), incluindo não somente atributos químicos, físicos e microbiológicos, mas também a macrofauna do solo, que desempenha um papel importante nestes estudos. Dos vários atributos analisados, somente os grupos Diplopoda, Chilopoda, Isoptera, biomassa total da macrofauna e índice de Shannon aplicado à macrofauna foram responsáveis por praticamente toda a separação entre as áreas, sendo os "bons" (Tabela I) indicadores das perturbações 
TABELA IV. Classes de indicadores de qualidade do solo definidas a partir do valor da taxa de discriminação paralela (TDP) resultante da análise canônica discriminante (ACD) quanto as funções canônicas 1 e $2\left(\mathrm{FCD}_{1} \mathrm{e}\right.$ $\mathrm{FCD}_{2}$ ), para cada atributo em áreas com araucária sob diferentes estados de conservação, independente do tratamento, na região de Campo do Jordão, SP.

\begin{tabular}{ccl}
\hline Valor de TDP do atributo & Classe de qualidade & Valor indicador \\
\hline$\leq 0,03^{*}$ & I & Baixo \\
$0,04-0,09$ & II & Médio \\
$0,10-0,20$ & III & Bom \\
$0,21-0,41$ & IV & Muito Bom \\
$0,42-0,80$ & V & Ótimo \\
$>0,81$ & VI & Excelente \\
\hline
\end{tabular}

*Valores podem variar de acordo com o ecossistema amostrado e o número de atributos químicos, físicos e biológicos do solo incluídos no modelo.

antrópicas que ocorrem nas áreas com araucária. Das variáveis ambientais, somente a matéria seca total da serapilheira, teores de $\mathrm{P}$ e os atributos microbiológicos, especialmente carbono da biomassa microbiana e respiração basal, contribuíram efetivamente para separar as áreas. Portanto, os resultados confirmaram a utilidade de diversos parâmetros relacionados com o carbono, especialmente o CBM, como bons indicadores da qualidade biológica do solo (Wardle \& Hungria 1994, Baretta et al. 2005, Maluche-Baretta et al. 2006). Além disso, a utilidade dos diplópodes (Diplopoda) e das aranhas (Araneae) como indicadores de perturbação e qualidade do hábitat (Warren \& Zou 2002) também foi confirmada.

Embora a densidade relativa (Indiv. $\mathrm{m}^{-2}$ ) de Oligochaeta (minhocas) tenha sido baixa nas coletas de setembro (2004) e fevereiro (2005), o que levou a um baixo valor de TDP, com o método TSBF (Tabelas I e II), este grupo merece uma atenção especial por ser considerado bom indicador de qualidade do solo (Paoletti \& Bressan 1996, Paoletti 1999, Baretta et al. 2007b). Para estudos futuros, recomenda-se a utilização de monólitos maiores $(40 \times 40 \mathrm{~cm})$ e formaldeído diluído $(0,5 \%)$ (Formol), garantindo assim, uma coleta rápida de espécies ativas na superfície (Amynthas, Urobenus) e dentro do solo (Glossoscolex spp.) conforme reportado por Baretta et al. (2007b).

Do ponto de vista prático, a aplicação do coeficiente TDP, que é resultado da $\mathrm{ACD}$, demonstrou ser uma poderosa ferramenta para a escolha de indicadores de qualidade do solo, pois indica quais são os principais atributos biológicos responsáveis pela separação entre as áreas, sendo estes considerados "bons" indicadores das perturbações antrópicas que ocorrem nas áreas com araucária. Seria desejável complementar estes estudos por meio da inclusão de outros atributos químicos, físicos e biológicos do solo no modelo da $\mathrm{ACD}$ e medir estes parâmetros com vários métodos de coleta em outros ecossistemas sob diferentes estados de conservação (Baretta et al. 2007a, b), a fim de validar potencial indicador de cada atributo edáfico. 


\section{AGRADECIMENTOS}

Os autores agradecem aos integrantes do Departamento de Ciência do Solo (ESALQ/ USP). Também agradecem ao CNPq (bolsa pesquisador) e ao programa BIOTA/FAPESP (www.biotasp.org.br), pelo financiamento do projeto temático (processo $\mathrm{n}^{\circ}$. 01/05146-6) e bolsa de Pós-Doutorado de Dilmar Baretta (processo nº . 2007/056376). Os autores agradecem a ajuda do Professor Winston Rios Ruiz pela correção do resumo em Espanhol.

\section{LITERATURA CITADA}

Alef, K. \& P. Nannipieri. 1995. Methods in applied soil microbiology and biochemistry. Academic Press, London.

Anderson J. M. \& J. S. I. Ingram. 1993. Tropical soil biology and fertility, a handbook of methods, $2^{\text {nd }}$ ed. CAB International, Wallingford.

Anderson, T. H. \& K. H. Domsch. 1993. The metabolic quotient for $\mathrm{CO}_{2}\left(q \mathrm{CO}_{2}\right)$ as a specific activity parameter to assess the effects of environmental conditions, such as $\mathrm{pH}$, on the microbial biomass of forest soils. Soil Biology and Biochemistry. 25: 393-395.

Athias, F., G. Josens \& P. Lavelle. 1975. Traits généraux du peuplement animal endogé de la savane de Lamto (Côte d'Ivoire). Pp. 375-388. In: J. Vanek (Ed). Progress in soil zoology. Academia, Prague.

Bandeira, A. G. \& A. Y. Harada. 1998. Densidade e distribuição vertical de macroinvertebrados em solos argilosos e arenosos na Amazônia Central. Acta Amazônica. 28: 191-204.

Baretta D., J. C. P. Santos., S. R. Figueiredo \& O. Klauberg-Filho. 2005. Efeito do monocultivo de pinus e da queima do campo nativo em atributos biológicos do solo no planalto sul catarinense. Revista Brasileira de Ciência do Solo. 29: 715-724.

Baretta, D., A. D. Brescovit., I. Knysak \& E. J. B. N. Cardoso. 2007a. Trap and soil monolith sampled edaphic spiders (Arachnida: Araneae) in Araucaria angustifolia forest. Scientia Agricola. 64: 375-383.

Baretta, D., A. L. Mafra., J. C. P. Santos., C. V. T. Amarante \& I. Bertol. 2006. Análise multivariada da fauna edáfica em diferentes sistemas de preparo e cultivo do solo. Pesquisa Agropecuária Brasileira. 41: 1675-1679.

Baretta, D., C. R. D. M. Maluche-Baretta \& E. J. B. N. Cardoso. 2008. Análise multivariada de atributos microbiológicos e químicos do solo em florestas com Araucaria angustifolia. Revista Brasileira de Ciência do Solo. 32: 2683-2691.

Baretta, D., G. G. Brown., S. W. James \& E. J. B. N. Cardoso. 2007b. Earthworm populations sampled using collection methods in Atlantic forests with Araucaria angustifolia. Scientia Agricola. 64: 384-392.

Brown G. G. \& C. Fragoso. 2003. El uso de la macrofauna edáfica en la agricultura del siglo XXI: problemas y perspectivas. Pp. 11-19. In: G. G. Brown, C. Fragoso \& L. J. Oliveira (Eds.). O uso da macrofauna edáfica na agricultura do século XXI: a importância dos engenheiros do solo. Embrapa Soja Série Documentos No. 224, Londrina.

Cochran, V. L., S. D. Sparrow \& E. B. Sparrow. 1994. Residue effects on soil micro and macroorganisms. Pp. 163-184. In: P. W. Unger (Ed). Managing agricultural residues. CRC Press, Boca Raton.

Cruz-Castillo, J. G., S. Ganeshanandam, B. R., Mackay, G. S. Lawes., C. R. O. O. Lawoko \& D. J. Woolley. 1994. Applications of canonical discriminant analysis in horticultural research. HortScience. 29: 1115-1119. 
Decaëns, T \& J. P. Rossi. 2001. Spatio-temporal structure of earthworm community and soil heterogeneity in a tropical pasture. Ecography. 24: 671-682.

Decaëns, T., T. Dutoit., D. Alard \& P. Lavelle. 1998. Factors influencing soil macrofaunal communities in post-pastoral successions of western France. Applied Soil Ecology. 9: 361-367.

Hair, J. F., R. E. Anderson \& R. L. Tatham. 1987. Multivariate data analysis with readings, $2^{\text {nd }}$ ed. Macmillan, New York.

Kladivko, E.J. 2001. Tillage systems and soil ecology. Soil and Tillage Research. 61: 61-76.

Kronka, F. J. N., M. A. Nalon, C. K. Matsukuma, M. M. Kanashiro, M. S. S. Ywane, M. Pavão, G. Durigan, L. P. R. Lima, J. R. Guillaumon, J. B. Baitello, S. C. Borgo, L. A. Manetti, A. M. F. Barradas, J. C. Fukuda, C. N. Shida, C. H. B. Monteiro, A. A. S. Pontinhas, G. G. Andrade, O. Barbosa, A. P. Soares, C. A. Joly \& H. T. Z. Couto. 2005. Inventário florestal da vegetação natural do Estado de São Paulo. Secretaria do Meio Ambiente, Instituto Florestal, São Paulo.

Lavelle, P., T. Decaëns, M. Aubert, S. Barot, M. Blouin, F. Bureau, P. Margerie \& J-P. Mora. 2006. Soil invertebrates and ecosystem services. European Journal of Soil Biology. 42: S3-S15.

Lima, H. V., T. S. Oliveira, M. M. Oliveira, E. S. Mendonça \& P. J. B. F. Lima. 2007. Indicadores de qualidade do solo em sistemas de cultivo orgânico e convencional no Semi-árido Cearense. Revista Brasileira de Ciência do Solo. 31: 1085-1098.

Maluche-Baretta, C. R. D., C. V. T. Amarante \& O. Klauberg-Filho. 2006. Análise multivariada de atributos do solo em sistemas convencional e orgânico de produção de maças. Pesquisa Agropecuária Brasileira. 41: 1531-1539.

Moço, M. K., E. F. Gama-Rodrigues, A. C. Gama-Rodrigues \& M. E. F. Correia. 2005. Caracterização da fauna edáfica em diferentes coberturas vegetais na região Norte Fluminense. Revista Brasileira de Ciência do Solo. 29: 555-564.

Odum, E. P. 1983. Ecologia. Guanabara, Rio de Janeiro.

Paoletti, M. G. \& M. Bressan. 1996. Soil Invertebrates as bioindicators of human disturbance. Critical Reviews in Plant Sciences. 15: 21-62.

Paoletti, M. G. 1999. Using bioindicators based on biodiversity to assess landscape sustainability. Agriculture Ecosystems \& Environment. 74: 1-18.

Raij B. V., J. C. Andrade, H. Cantarella \& J. Á. Quaggio. 2001. Análise química para avaliação da fertilidade de solos tropicais. Instituto Agronômico, Campinas.

Stork, N. E. \& P. Eggleton. 1992. Invertebrates as determinants and indicators of soil quality. American Journal of Alternative Agriculture. 7: 38-47.

ter Braak, C. J. F. \& P. Smilauer. 1998. CANOCO reference manual and user's guide to Canoco for Windows: software for canonical community ordination (version 4). Microcomputer Power, New York.

Vance, E. D., P. C. Brookes \& D. S. Jenkinson. 1987. An extraction method for measuring microbial biomass C. Soil Biology and Biochemistry. 19:703-707.

Wardle, D. A. \& M. A. Hungria. 1994. A biomassa microbiana do solo e sua importância nos ecossistemas terrestres. Pp. 193-216. In: R. S. Araújo and M. Hungria (Eds). Microrganismos de importância agrícola. Embrapa, Brasília.

Warren, M. W. \& X. Zou. 2002. Soil macrofauna and litter nutrients in three tropical tree plantations on a disturbed site in Puerto Rico. Forest Ecology and Management. 170: 161-171. 